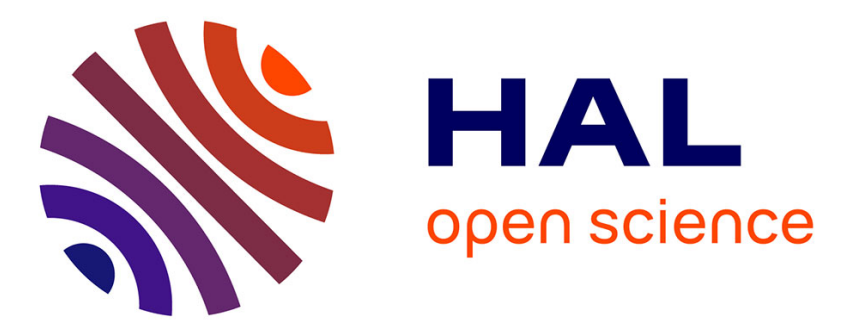

\title{
Paving the way for multiple applications for the 3D-AFM technique in the semiconductor industry
}

\author{
J. Foucher, E. Pargon, M. Martin, S. Reyne, C. Dupré
}

\section{To cite this version:}

J. Foucher, E. Pargon, M. Martin, S. Reyne, C. Dupré. Paving the way for multiple applications for the 3D-AFM technique in the semiconductor industry. SPIE 2008, 2008, San Jose, United States. pp.6922, $10.1117 / 12.772675$. hal-00384223

\section{HAL Id: hal-00384223 \\ https://hal.science/hal-00384223}

Submitted on 14 Mar 2020

HAL is a multi-disciplinary open access archive for the deposit and dissemination of scientific research documents, whether they are published or not. The documents may come from teaching and research institutions in France or abroad, or from public or private research centers.
L'archive ouverte pluridisciplinaire HAL, est destinée au dépôt et à la diffusion de documents scientifiques de niveau recherche, publiés ou non, émanant des établissements d'enseignement et de recherche français ou étrangers, des laboratoires publics ou privés. 


\title{
Paving the way for multiple applications for the 3D-AFM technique in the semiconductor industry
}

\author{
J.Foucher $^{a}$, E.Pargon ${ }^{b}$ M.Martin ${ }^{b}$, S.Reyne ${ }^{a}$, C.Dupré $^{a, c}$ \\ aCEA-LETI-MINATEC, 17 avenue des Martyrs, 38054 Grenoble Cedex 9, France johann.foucher@cea.fr \\ ${ }^{b}$ LTM/CNRS, 17 avenue des Martyrs, 38054 Grenoble Cedex 9, France \\ ${ }^{\mathrm{I}}$ IMEP-LAHC, INPG-MINATEC
}

\begin{abstract}
The 3D-AFM technique is a very well known technique as a non destructive reference to calibrate CD-SEM and Scatterometry metrology. However, recent hardware, tip design and tip treatment improvements have offered to the technique new capabilities that pave the way for multiple applications in the semiconductor industry. The 3D-AFM technique is today not only a calibrating technique but also a process control technique that can be use either at the R\&D level or in fab environment.

In this paper, we will address the limits of the 3D-AFM technique for the semiconductor industry depending on the applications by focusing our study on tip to sample interactions. We will identify, test and validate potential industrial solutions that could extend the 3D-AFM potentialities. Subsequently, we will show some interesting applications of the technique related to LER/LWR transfer during silicon gate patterning and related to advance multiwires devices fabrication.
\end{abstract}

Keywords: CD, 3D-AFM, tip wear, interaction, capabilities, accuracy, LER, LWR

\section{INTRODUCTION}

The 3D-AFM technique is a derived technique from conventional and very well-known AFM technique ${ }^{1}$. The discovery of the AFM technique in 1986 by Binnig, Quate and Gerber, has demonstrated the capability of the technique to investigate conductive and insulator materials with high sensitivity to measure forces as small as $10^{-18} \mathrm{~N}$ and based on tunneling detection of the lever deflection. Since then, many improvements have been done related to this technique. Martin and Wickramasinghe demonstrate the capability of the AFM technique to investigate surfaces with high sensitivity based on optical detection of the lever deflection ${ }^{2}$. It is only in 1994 that Martin and Wickramasinghe invented the CD-AFM ${ }^{3}$ and demonstrate the capability of the technique to image pattern sidewall through the combination use of flared silicon tip and local surface slope detection in 2 dimensions. Afterwards, we had to "wait" until 2005 in order to see high order tip effects that are taken into account during tip shape extraction ${ }^{4}$ after measuring the pattern. In 2007, scan enhancements have lead the CD-AFM technique to really become the 3D-AFM technique by reducing drastically the sidewall noise level and by removing the notching data artifact at the bottom of measured patterns $^{5}$. As an immediate result, the lateral resolution (or profile resolution) of the technique has reached today the nanometer level $^{6}$ (cf. figure 1).

Today, there is a special need for accuracy in most of the semiconductor industry fields. Indeed, the continuous scaling down of patterns has reached such level that conventional industrial CD metrology techniques (such as CD-SEM and Scatterometry...) are very challenged in term of accuracy. Therefore, CD metrology tool suppliers request for accurate tool calibration and the semiconductor industry increasingly requests also for accuracy in their production CD standards (golden wafers) calibration and requests for accuracy in their process development metrology such as OPC control, process window determination based on FEM (...). The 3D-AFM technique has a key role to play in order to fulfill on time advanced roadmap recommendations in order to reduce $\mathrm{R} \& \mathrm{D}$ and Production costs. The success of the 3D-AFM technique relies on its ability to reach sub-1nm accuracy. This level of accuracy relies on: 1Scanning code enhancement; 2- Servoing quality; 3- Tip design;

\begin{tabular}{|c|c|}
\hline YEAR & PROFILE (or LATERAL) RESOLUTION \\
\hline $1986-1987$ & $\begin{array}{c}\text { Very poor due to the combined use of } \\
\text { conical tip and no lateral servo }\end{array}$ \\
\hline 1994 & $>10 \mathrm{~nm}$ (simple tip width removal) \\
\hline 2005 & $3 \mathrm{~nm}$ \\
\hline 2008 & $1-2 \mathrm{~nm}$ \\
\hline
\end{tabular}

Figure 1: Profile resolution or lateral resolution evolution of the AFM technique

4-Tip shape characterization; 5- Tip shape extraction and 6: Tip wear control. 
In this paper, we will address this last item ("the tip wear control") by presenting an advanced study illustrating the main origin of the tip wear related to the use of standard flared silicon tip and the potential solution in order to avoid this wear. This study is based on physical and chemical surface and bulk materials characterization based on XPS, FTIR, ATR and Contact Angle analysis techniques. We will conclude with various new promising applications of the 3D-AFM technique that open the way for multiple applications of the technique for the semiconductor industry such as LER/LWR mechanisms transfer during plasma etching and a new promising multiwires device application.

\section{EXPERIMENTAL SET-UP}

In this paper, the 3D-AFM equipment used is the Dimension X3D from Veeco which has been well described in other papers ${ }^{7,8,9}$. The flared silicon tip used is the CDR70 model from Team Nanotec GmbH which corresponds to a 70nm diameter tip. Concerning the advanced tip wear study, the analysis techniques used are X-Ray Photoelectron Spectroscopy (XPS), Fourier Transform Infrared (FTIR) Spectroscopy, Attenuated Total Reflection (ATR) and Contact Angle measurement.

\section{3D-AFM ADVANCED STUDY}

\subsection{CONTEXT OF THE STUDY}

At the origin of this study, we would like to simply use the benefit of using the 3D-AFM technique in order to benchmark different resists for implant lithography process development. The goal was to study and optimize standing waves on the final resist profile and study how the profile was impacted after an implant process step. As illustrated in figure 2, after the lithography step, as there was no BARC layer we can clearly identify standing waves formation on the cross-section SEM view. After measuring it by 3D-AFM, we have validated that we were able to obtain the same level of accuracy without cleaving the wafer and therefore saving time and cost as illustrated in figure 3 that shows the 3D-AFM results on such resist profile. The tapered profile observed on figure 3 is just due to a non 1:1 scale. In this case, there was no issue with the 3D-AFM used as there was no tip wear and excellent data quality.

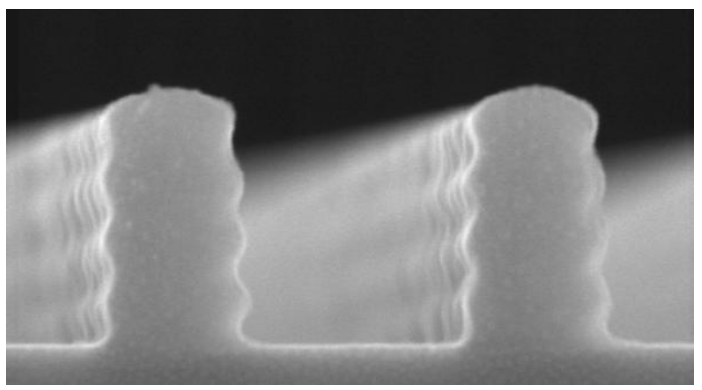

Figure 2 : SEM cross-section view of one type of 193 resist used for implant level lithography

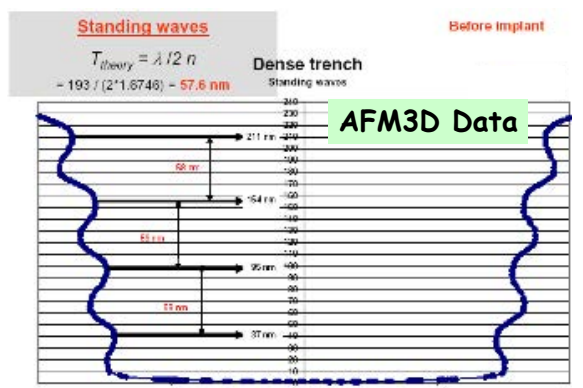

Figure 3 : 3D-AFM measurement of 193 resist without the use of a BARC layer

Afterwards, we applied an implant process on the resist and look at the new resist profile with the 3D-AFM technique in order to see how this type of resist was degrade during the process and if the new profile was acceptable for the following technological steps. The results are illustrated on figure 4. On a first look, we can clearly see that standing waves amplitude does not change during the implant process from $5 \mathrm{keV}$ up to $20 \mathrm{keV}$. However, the important point that we have to look at is represented on figure 5. Indeed, as we can clearly see on this figure, for a $40 \mathrm{keV}$ implant process, the tip wear level ( $20 \%$ diameter drop after only 5 measurements) is very important and has reached a level which is definitely not acceptable for the use of the 3D-AFM technique in a fab environment.

Based on this result, we decide to evaluate and quantify the modifications induced by implantation on resist surface. Subsequently, the final goal is to understand the reason why these new surface characteristics lead to changes in 3DAFM tip-surface interactions. 


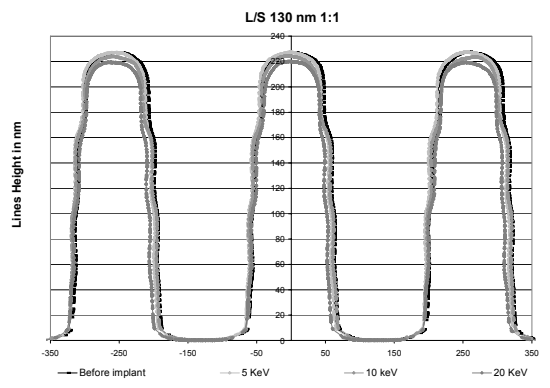

Figure 4: 3D-AFM measurements of resist profiles after an implant process for various energy levels

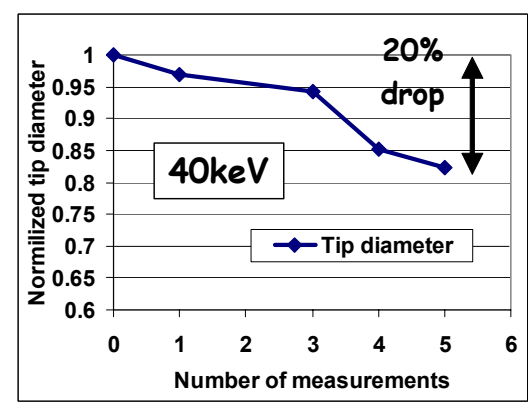

Figure 5: Normalized 3D-AFM flared silicon tip width variation as a function of number of measurements on resist profiles after applying a $40 \mathrm{keV}$ implant process

\subsection{PROTOCOL OF THE ADVANCED STUDY ON TIP WEAR ORIGIN}

Our reference resist for the study is a 193 resist (a Methacrylate matrix-based resist). We have compared two different implant process conditions: $1-40 \mathrm{keV} / 8.5 \mathrm{E} 14 \mathrm{at} / \mathrm{cm}^{2}$ and $2-10 \mathrm{keV} / 5 \mathrm{E} 13 \mathrm{at} / \mathrm{cm}^{2}$. After the evaluation of all the modifications on surface that would be responsible for 3D-AFM tip damaging, we will correlate data from the different analysis methods and afterwards try to find a tip coating allowing reliable measurements with the $3 \mathrm{D}-\mathrm{AFM}$ technique in order to implement it potentially in a production environment.

For this specific study, we decide to move from patterned wafers to blanket wafers in order to allow the chemical surface analysis with XPS, FTIR, ATR and contact angle measurements on the same wafers. The figure 6 summarizes the measurement capability of each technique. We have divided the study in 3 parts, extreme surface analysis (XPS and Contact angle), surface analysis (ATR) and volume analysis (FTIR). As a reminder, the figure 7 represents the typical chemical compositions of a 193 resist based on a methacrylate matrix. Each group that has been represented on this figure play a key role in the resist behavior related to lithography performance, the etching selectivity during plasma etching, resist adhesion on substrate (...). In our study we will identify and follow the behavior of each group.

\subsection{RESULTS}

\subsubsection{Reference measurements}

We have started to acquire reference data from each technique in order to have access to the chemical "signature" of the initial 193 resist before applying the implant process. The presented FTIR and ATR spectra are only data extracted from the full spectrum. The figure 8 and 9 show respectively the XPS and FTIR/ATR signatures of the 193 resist.

We can clearly see on figure 8, that the model choose (Methacrylate model, blue curve), for our XPS analysis fit, is in keeping with the experimental data (red curve). On the figure 9, we can clearly identify all the main groups' constituents of our 193-nm resist. 


\section{3-nm resist: Reference XPS}

-193-nm resist based on Methacrylate matrix

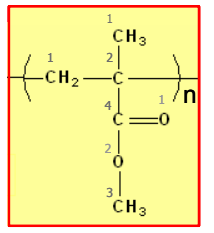

- Methacrylate model applied to our 193-nm resist

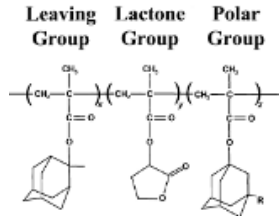

MAMA/ $\alpha$-GBLMA/RAMA

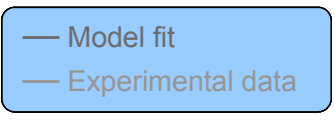

\begin{tabular}{lccccccc}
\multicolumn{3}{c}{ methacrylate } & \multicolumn{3}{c}{ C 1s } & & \multicolumn{3}{c}{ O 1s } \\
\cline { 2 - 4 } \cline { 7 - 8 } & 1 & 2 & 3 & 4 & & 1 & 2 \\
\hline BE (eV) & 285,00 & 285,72 & 286,79 & 289,03 & & 532,21 & 533,77 \\
FWHM (eV) & 1,15 & 1,06 & 1,28 & 0,99 & & 1,27 & 1,39 \\
Area (\%) & 42 & 21 & 21 & 17 & & 51 & 49 \\
Area (\%) & 0,13 & 0,00 & 0,12 & 0,10 & & 0,10 & 0,12 \\
$\boldsymbol{m}$ & 0,88 & 0,74 & 0,62 & 0,98 & & 0,79 & 1,00 \\
\hline
\end{tabular}
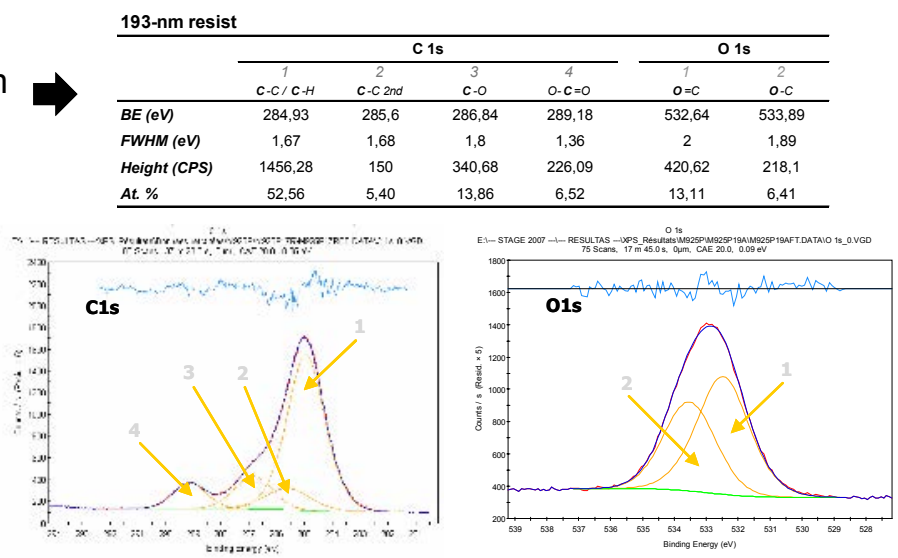

Figure 8: XPS reference data on our 193-nm resist before the implant process

\section{3-nm resist: Reference FTIR and ATR}

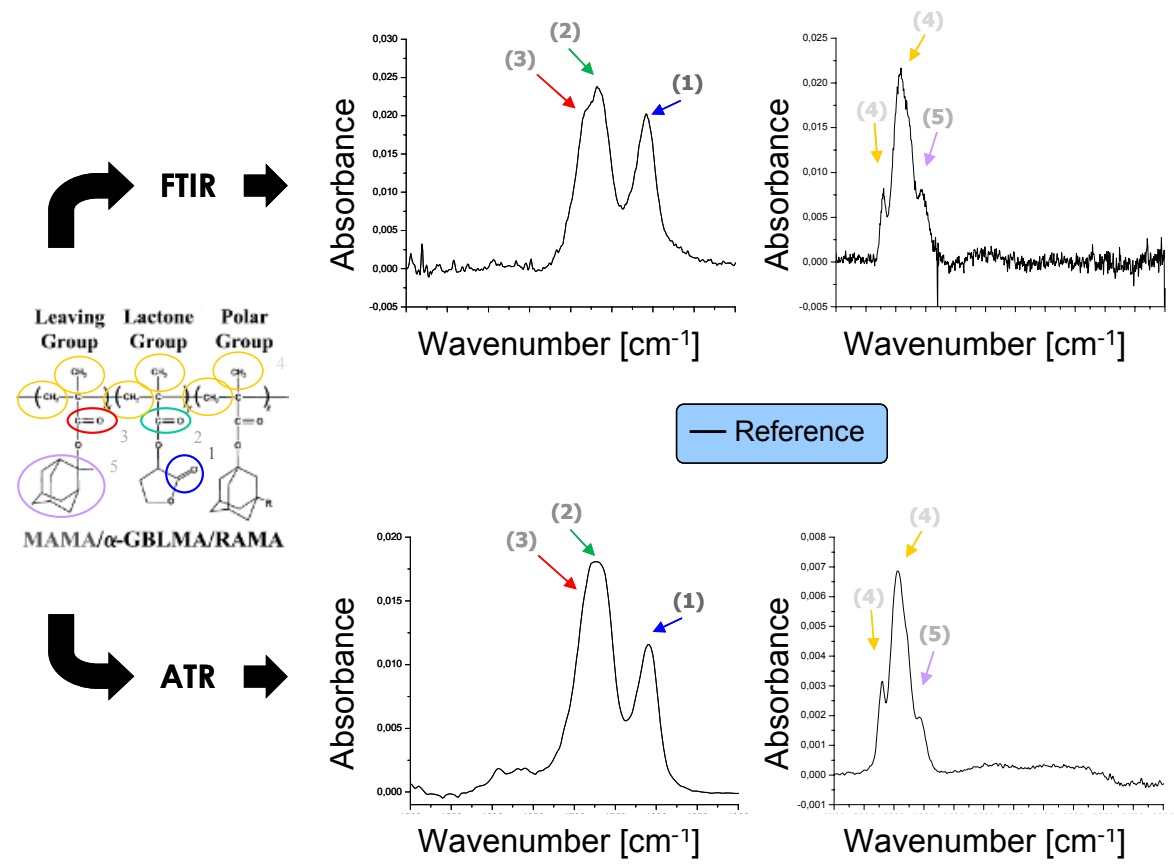

Figure 9: FTIR and ATR reference data on our 193-nm resist before the implant process 


\subsubsection{Measurements after implant processes at $10 \mathrm{keV}$ and $40 \mathrm{keV}$}

\section{$\underline{\text { XPS }}$}

The figure 10 shows the O1s peak after XPS analysis. The Reference and the two implant conditions are compared on this figure. The Implant 1 condition induces a soft shift to lower binding energy. It seems that eventually other type of $\mathrm{O}$ bonding (i.e $\mathrm{H}_{2} \mathrm{O}$ or $\mathrm{OH}$ ) has appeared. However the implant 2 has no impact on the O1s bindings.
Implant: 40keV vs. 10keV - XPS

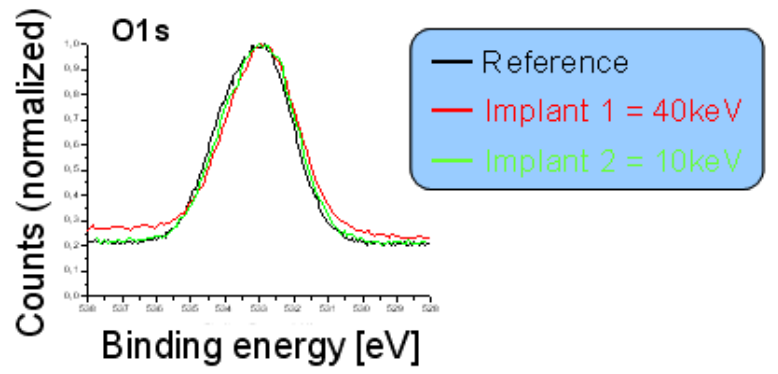

Figure 10: XPS data analysis comparing the reference and the two implant process conditions for the O1s peak

\section{FTIR and ATR}

The figure 11 shows the results obtained with FTIR and ATR techniques and compares the reference to the two implant conditions. The thing that is very important in our case to focus on is the $\mathrm{H}_{2} \mathrm{O} /-\mathrm{OH}$ peak that appears on both FTIR and ATR results. It confirms our initial hypothesis makes after the XPS results that the 193-nm resist which is initially relatively hydrophobic seems to become hydrophilic after the $40 \mathrm{keV}$ implant process condition because of $\mathrm{H}_{2} \mathrm{O}$ adsorption.

On the basis of this result, we have carried out some contact angle measurements in order to confirm the trend observed in the case of the two process conditions.

\section{CONTACT ANGLE}

Polymers are low compact material with low surface free energy, generally ranging from 10 to $50 \mathrm{mN} / \mathrm{m}$ (assuming a repeatability of $2-3 \mathrm{mN} / \mathrm{m}$ ). The figure 12 shows the results coming from contact angle measurements and comparing the reference to the two implant process conditions. We can clearly see that in the case of the implant 1 condition, the surface is drastically modified as the surface free energy increases both for the dispersive and polar parts. However, the implant 2 condition does not show any increase and the polar part tends to decrease compare to the reference.
Implant: 40keV vs. 10keV - FTIR and ATR
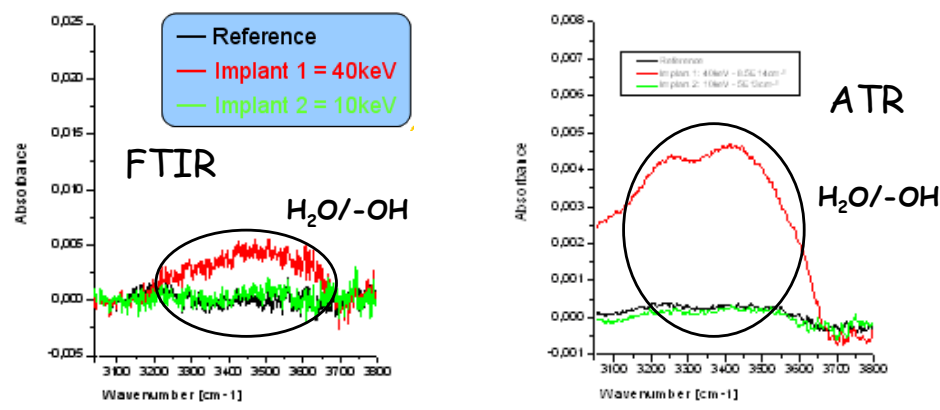

Figure 11: FTIR and ATR data analysis comparing the reference and the two implant process conditions and showing the presence of

$$
\mathrm{H}_{2} \mathrm{O} /-\mathrm{OH}
$$

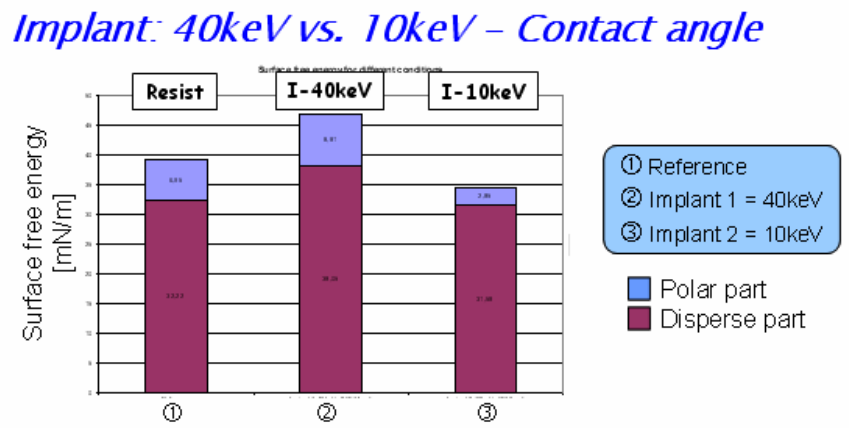

Figure 12: Contact angle data analysis comparing the reference and the two implant process conditions 
Finally we can clearly conclude that the main origin of the premature wear of flared silicon tip used in this particular case seems to be the hydrophilicity of the sample during measurements which leads to higher sticking coefficient of the tip. This idea related to the use of the 3D-AFM technique has been previously mentioned in Liu et $\mathrm{al}^{10}$ work but they do not demonstrate anything. It is the case now based on our work. In order to confirm this hypothesis, we have developed our homemade hydrophobic conformal coating. The figure 13 shows the results obtain with this hydrophobic treatment by measuring $193-\mathrm{nm}$ resist after a $40 \mathrm{keV}$ implant process. The tip diameter evolution is compared to the first result obtain with a conventional flared silicon tip.

This conclusion and potential industrial solution open the way for multiple applications for the 3D-AFM technique in the semiconductor industry as one the main issue (the tip wear) could be solved.
Results after tip coating for implant process

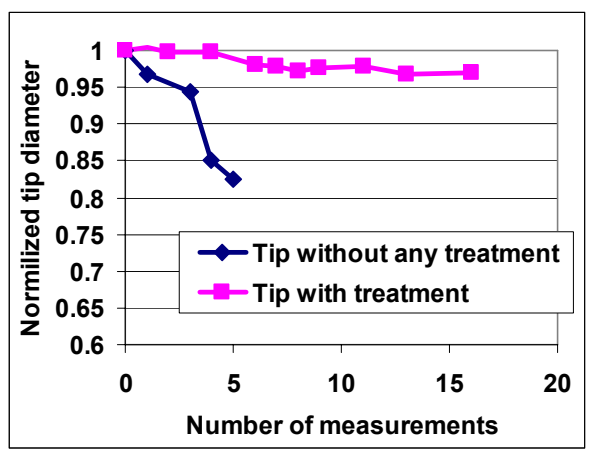

Figure 13: Tip diameter evolution with and without hydrophobic coating as a function of the number of measurements on a 193-nm resist previously exposed to an implant process at $40 \mathrm{keV}$ energy.

\section{LER/LWR TRANSFER DURING PLASMA ETCHING FOR ULTIMATE PATTERNING}

As an illustration of this work, we have started to work on LER/LWR transfer during plasma etching for ultimate gate patterning. The measurement protocol for this work is presented on figure 14. It consists of using the 3D-AFM technique and its ability to track sidewall information in order to extract LER and LWR information after each technological step involved in gate patterning. In our case, the studied material stack is quite simple and consists in 193 resist, BARC layer and Poly-Silicon layer for the final transistor gate definition.

The step 1 representing the initial $193-\mathrm{nm}$ resist LWR measurement is quite simple to carry out with the 3D-AFM technique. Typically, before carrying out the step 2 that consists in opening the BARC layer, we have to improve the 193$\mathrm{nm}$ resist selectivity to plasma etching by applying an $\mathrm{HBr}$ plasma (so called: cure process) otherwise the transfer of the pattern

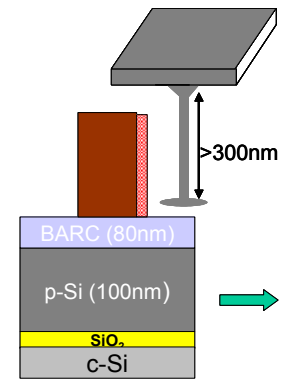

1)Lithography

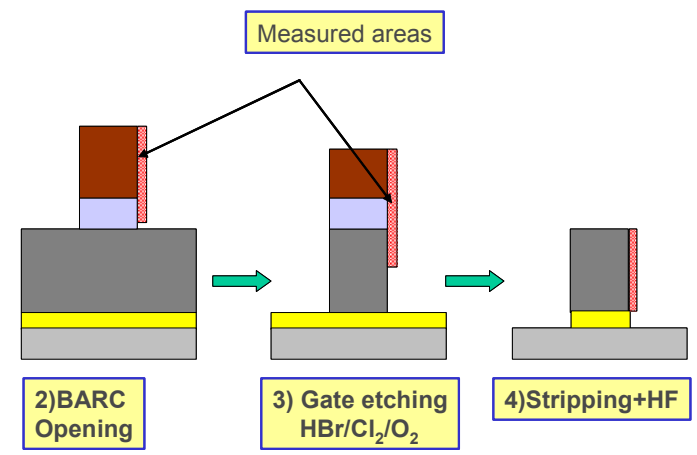

Figure 14: 3D-AFM protocol used to study LER/LWR transfer during silicon gate patterning into the final gate could be difficult for ultimate patterning. We have therefore carried out some LWR measurement after $\mathrm{HBr}$ plasma. The figure 15 represents the tip diameter evolution as a function of the number of measurements and related process applied for each measurement. It consists in the measurement of 193 resist first and then the same 193 resist after a cure process. We can clearly see that when applying $\mathrm{HBr}$ plasma we had particle contamination of our tip which subsequently makes impossible the advanced LWR study and leads to high cost per measurement. Therefore, we have applied our hydrophobic coating on our silicon tip in order to see if it could also solve the particle contamination issue. The figure 16 shows the results obtain in term of tip diameter evolution as a function of the number of measurements done on 193 resist after applying only $\mathrm{HBr}$ plasma.

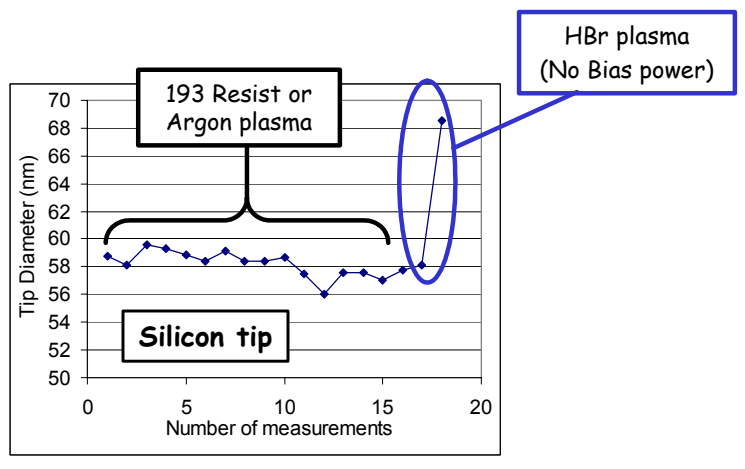

Figure 15: Tip diameter evolution as a function of the number of measurements and process conditions 
We can clearly see that the hydrophobic coating here again has been very efficient to solve this problem of particle contamination after an $\mathrm{HBr}$ plasma process. Based on this result we have continued to study various plasma chemistries that are summarized on the figure 17 for which we had some trouble with particle contamination or tip premature wear. As it is illustrated in the $2^{\text {nd }}$ column on figure 17 , when using the tip coated (called ASL which stands for Anti-sticking layer) with hydrophobic layer, most of the plasma chemistries that used to present some issues have been solved except for two of them. We assume that another wear mechanism of the tip could be complementary to the hydrophilicity of the sample.

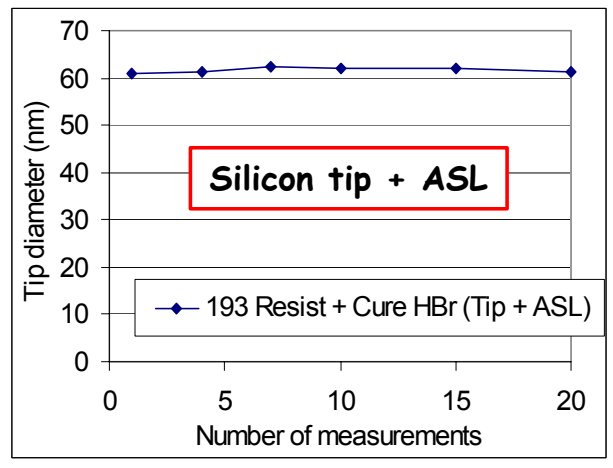

Figure 16: Tip diameter evolution as a function of the number of measurements on 193 resist after $\mathrm{HBr}$ plasma process

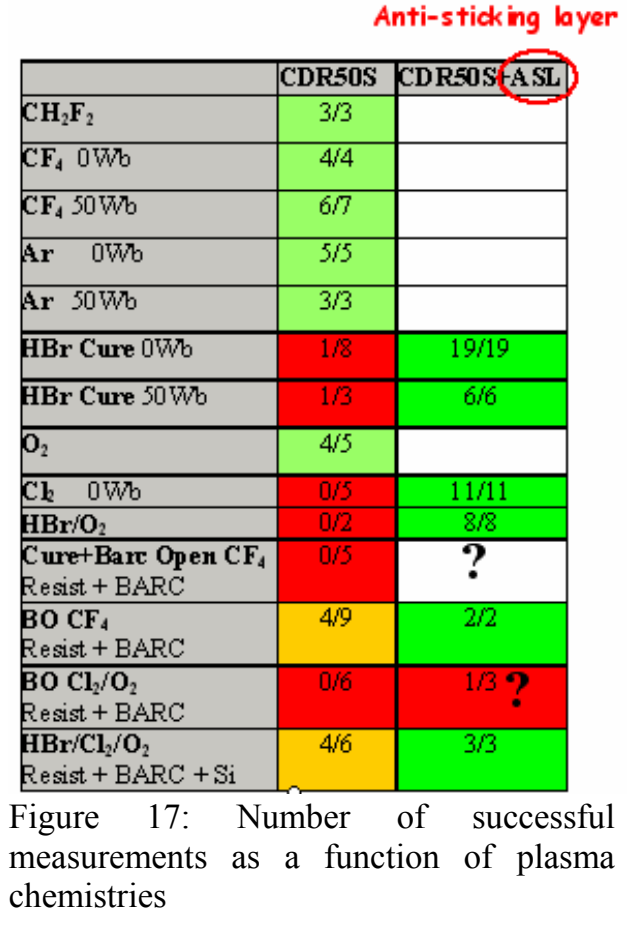

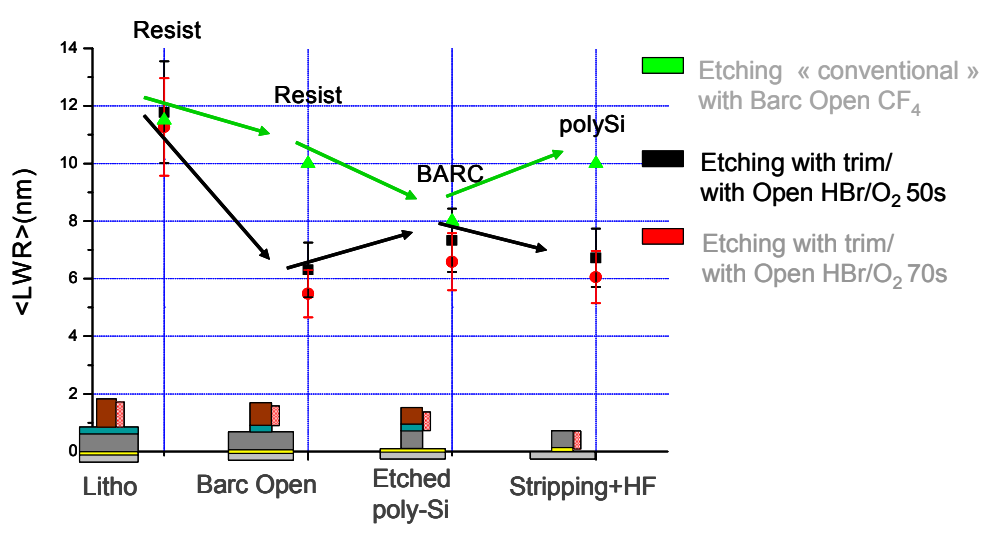

Figure 18: LWR transfer from the lithography step down to the silicon gate definition. The influence of a plasma resist trimming process is presented

The figure 18 illustrates one of the final result obtains with such LWR study. This graphic represents the LWR evolution as a function of the technological step applied on a wafer in order to design a silicon gate based on the top-down approach. In addition to the successive technological step, we have compared the LWR transfer with and without a 193 resist trimming process. Such plasma process is very useful in order to make smaller devices and therefore by-pass ultimate lithography resolution that could not be currently achieved. We can clearly see on this graphic that for both processes (with and without resist trimming), the final silicon gate LWR is the same value than the one of the BARC LWR. If we now compare with and without a resist trimming process, we can clearly see that without it, the LWR reduction between the initial 193 resist and the final silicon gate is around 15\% whereas with this specific resist trimming process the reduction has reached $45 \%$ and remain at this same level on the final gate.

\section{ADVANCED APPLICATION FOR NEW PROMISING MULTIWIRES DEVICES FABRICATION}

After exploring and extending the potential applications of the 3D-AFM technique to fundamental plasma etching studies and implant process studies, we would like to combine both complex structure measurement and validate on it 
the capability of the 3D-AFM technique to reach the nanometer resolution. In order to carry out this experiment, we have processed new promising multiwires device wafer as it shown on the figure 19 on the first cross-section image. We can clearly see the three different SiGe wires with void between each. The final goal in a near future is to control the sidewall roughness of the wires, control the width and the shape in the range of $20 \mathrm{~nm}$ and once they are fabricated, the goal is to control within few nanometers of accuracy the Si epitaxy on these SiGe wires. The useful devices have wires with a length of 250nm. Typically, in order to control the process, engineers are doing cross-section images and In-line TEM analysis on longer wires because the sample preparation is impossible on only $250 \mathrm{~nm}$ wire length. Our goal is therefore to validate the capability of the $3 \mathrm{D}-\mathrm{AFM}$ to control the overall process fabrication on real devices with a $250 \mathrm{~nm}$ length and faster than with these two techniques in order to reduce R\&D cost.

The figure 19 shows the 3D-AFM results. We have validated the capability to measure the wires with a length of $250 \mathrm{~nm}$. On the average 3D-AFM profile (blue curve), we can clearly identify all the wires that present a width in the range $25 \mathrm{~nm}$ as it has been measured with TEM analysis. On the final graphic which is the superimposing of the 3D-AFM wires measurements before and after the Silicon epitaxy on the SiGe wires, we have validated the capability of the 3D-AFM technique to characterize such process at the exact same position before and after the epitaxy process which is strictly impossible with another current $\mathrm{CD}$ metrology technique.

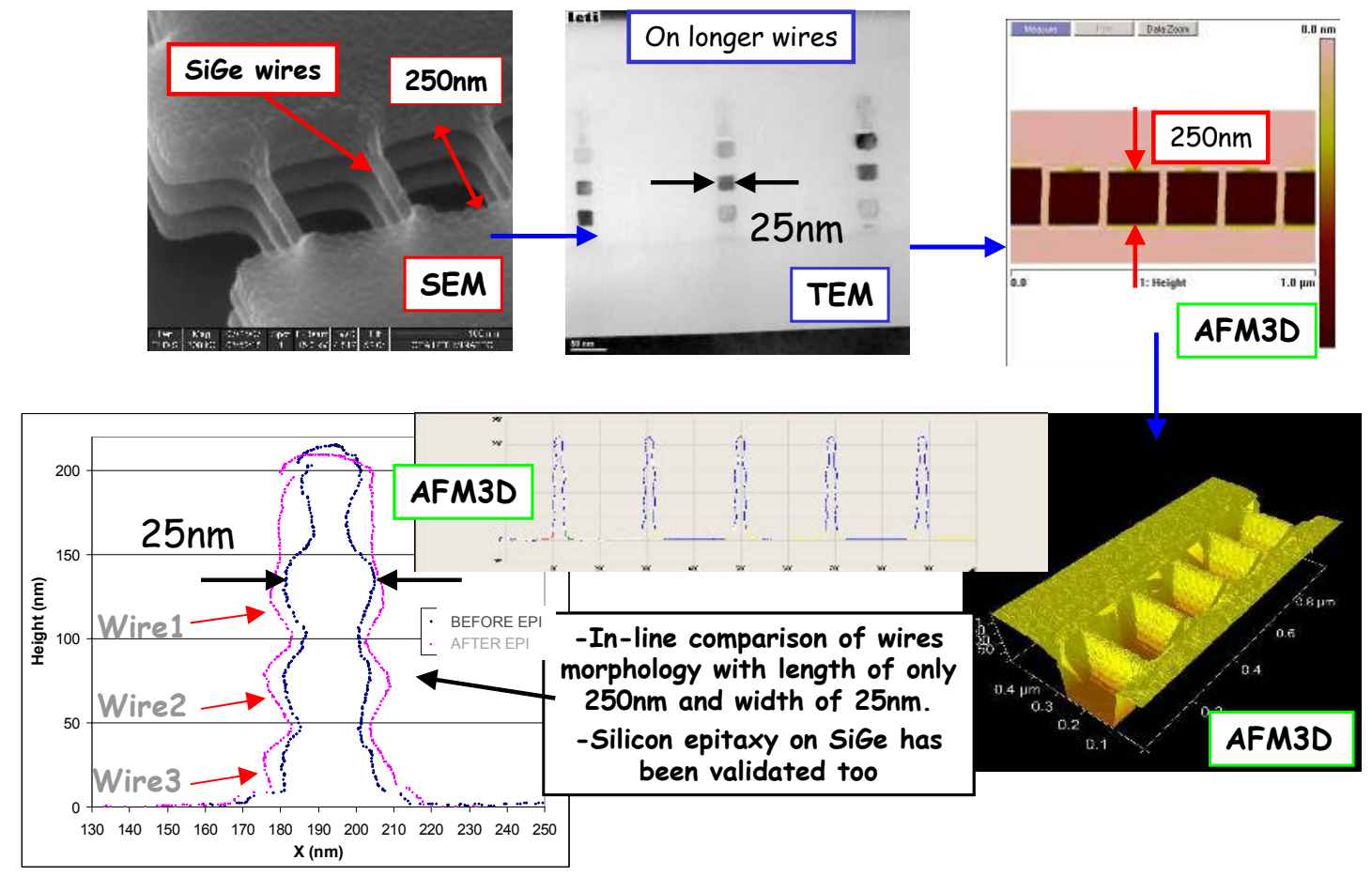

Figure 19: Example of a complex multiwires device that has been characterized with the 3D-AFM technique

\section{CONCLUSION}

The tip wear is a strong limitation for a massive introduction of the 3D-AFM technique for advanced process control in order to be complementary to Scatterometry and CD-SEM techniques and allow fab environment to get enough CD measurement accuracy compatible with roadmap requirements.

In this study we have shown that the hydrophilicity of samples is partly responsible of tip damage due to higher sticking coefficient (particle pick-up or tip erosion) that occurs during some implant process and during plasma etching processes. We have shown that hydrophobic coating realized on conventional flared silicon tips is the only way to overcome premature tip damage. However, it is not enough as we are still not able to measure for example resist pattern that have been etched with $\mathrm{Cl}_{2} / \mathrm{O}_{2}$ plasma chemistry. It opens the way to understand more deeply the tip to sample interaction. In any case, by using such hydrophobic tip, it has allowed us to work in advance on LER/LWR transfer during plasma etching processes for example by showing that applying a resist trimming process could reduce the initial 193 resist LWR by a factor of $45 \%$ on the final silicon gate. When combining such a tip with recent 3D-AFM 
enhancements, we have been able to fully characterize in advanced a new promising complex multiwires devices that currently no other in-line technique (either CD-SEM or Scatterometry) are able to measure.

The 3D-AFM industry has to work hard and fast on manufacturing such hydrophobic tips and continuing to improve tip servoing in order to help the semiconductor industry to reach the accuracy that is required by the ITRS and by all the other CD metrology techniques which require accurate calibration within $1 \mathrm{~nm}$.

\section{ACKNOWLEDGEMENTS}

The authors would like to thank the different persons involved in LETI multiwires devices fabrication (T.Ernst, V.Delaye, J-M.Hartmann, S.Pauliac, C.Vizioz). We would also like to thank A.Pikon (Rohm\&Haas) for providing 193 resist, cross-section images and related AFM3D data. Finally, we would like to thank all the Veeco R\&D team in Santa Barbara for fruitful discussions on the 3D-AFM technique.

\section{REFERENCES}

${ }_{1}^{1}$ «The Atomic Force Microscope »-G.Binnig, C.F.Quate and Ch.Gerber -Phys.Rev.Lett. 1985.

2 «AFM mapping and profiling on a sub-100Å scale»-Y.Martin, H.K Wickramasinghe, Appl.Phys.Lett. 1987.

3 « Method for imaging sidewalls by AFM»-Y.Martin, H.K Wickramasinghe, Appl. Phys. Lett. 1994.

4 « Tip Characterization and surface reconstruction of complex structures with CD-AFM » -G.Dahlen et al, J. Vac. Sci. Technol.B, 2005.

5 «TEM validation of CD-AFM image reconstruction »-G.Dahlen et al, Proc.SPIE Microlithography, 2007

${ }^{6}$ " Advances in CD-AFM scan algorithm technology enable improved CD Metrology »-L.Mininni, J.Foucher, Proc. SPIE Microlithography, 2007.

7 «Study of 3D metrology techniques as an alternative to cross-sectional analysis at the R\&D level» J.Foucher and K.Miller, Proc. SPIE53 75, 444 (2004).

${ }^{8}$ « From CD to 3D Sidewall Roughness Analysis with 3D CD-AFM», J.Foucher, Proc. SPIE 5752, 966 (2005).

9 «Minimizing CD Measurement Bias through Realtime Acquisition_of 3D Feature Shapes» J.Foucher, D.Gorelikov, M.Poulingue, P.Fabre, and G.Sundaram, Proc. SPIE 6152, 61521A (2006).

${ }^{10}$ « Advanced atomic force microscopy probes : Wear resistant designs »-H.Liu et al, J.Vac.Sci.Technol.B 23(6) 2005. 